\title{
Incidence of delirium after cardiac surgery: protocol for the DELIRIUM-CS Canada cross-sectional cohort study
}

\author{
The DELIRIUM-CS Investigators*; for the Canadian Cardiovascular Critical Care Society Investigator \\ Group and the Canadian Critical Care Trials Group
}

\section{Abstract}

Background: Delirium is a recognized complication of cardiac surgery and is the focus of increasing attention owing to its negative effect on postoperative outcomes. However, little is known about the actual incidence of delirium following cardiac surgery, with published rates ranging widely, from $3 \%-78 \%$. We describe the protocol for the DELIRIUM-CS Canada study, which will use validated and easily implementable bedside tools to determine the incidence of postoperative delirium in a contemporary cardiac surgery population. We hypothesize that delirium, identified through a systematic and standardized screening protocol, is a highly prevalent, though variable, condition following cardiac surgery.

Methods: The DELIRIUM-CS Canada study is a multicentre cross-sectional cohort study. Over a 3-month period, all patients undergoing major cardiac surgical procedures at 10 participating centres will be screened for postoperative delirium by means of the Intensive Care Delirium Screening Checklist or the Confusion Assessment Method for the Intensive Care Unit. Delirium screening will be conducted for 7 days following the date of surgery or until the initial discharge from the intensive care unit. In addition to reporting an overall rate of delirium, we will report unadjusted and adjusted incidence rates of delirium by institution and for the entire cohort. Risk adjustment will be performed with the use of multivariate regression modelling techniques.

Interpretation: The results of this study will provide valuable insight into the true burden of delirium among patients having undergone a major cardiac surgical procedure in the current era. This is the first step in creating a multifaceted delirium prevention/ treatment clinical pathway for patients undergoing cardiac surgery. Trial registration: ClinicalTrials.gov, no. NCT02206880.

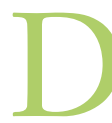

elirium is an acute confusional state characterized by fluctuating mental status, inattention, and disorganized thinking or altered level of consciousness. ${ }^{1-3}$ It has long been recognized as a complication of cardiac surgery and is more likely to be experienced by older adult patients and those with greater comorbid disease burden. ${ }^{4-7}$ Recently, attention has been paid to the negative effect of delirium on health care costs and postoperative outcomes, including long-term survival, hospital readmission rates, and reduced cognitive and functional recovery. ${ }^{8-12}$ Despite this, little is known about the true burden of delirium among patients having undergone a cardiac surgical procedure. Reported rates of the condition following cardiac surgery range widely, from as low as $3 \%$ to as high as $78 \% .{ }^{13}$ Delirium remains an underrecognized source of end-organ dysfunction. ${ }^{13}$ In the absence of an active screening program, it can go undiagnosed in more than $70 \%$ of cases. ${ }^{6,13,14}$
Over the past 2 decades, the risk profile of patients undergoing cardiac surgery has changed. Increasingly, cardiac surgical procedures are being performed in older and sicker patients who are at an increased risk for postoperative delirium. ${ }^{15}$ As such, rates of postoperative are likely to continue to increase owing to the aging of the population. Quantifying the magnitude of delirium in a large contemporary sample of the population is the first critical step in addressing the

Competing interests: None declared.

*The list of investigators appears at the end of the article.

Correspondence to: Rakesh Arora, rakeshcarora@gmail.com This article has been peer reviewed.

CMAJ Open 2017. DOI:10.9778/cmajo.20160136 
prevention and treatment of this life-threatening complication. The Canadian Cardiovascular Critical Care Society considers delirium following cardiac surgery a national priority requiring investigation.

We describe the protocol for the DELIRIUM-CS Canada study. The aims of the study are threefold: 1) to identify the true burden of delirium, using a standardized definition, among patients having undergone a cardiac surgical procedure in Canada, 2) to determine the impact on use of health care resources (as determined by intensive care unit [ICU] and hospital length of stay) and postoperative mortality and 3 ) to determine modifiable and nonmodifiable risk factors for the occurrence of delirium in a large multicentre cohort of contemporary patients having undergone cardiac surgery. We hypothesize that delirium is a highly prevalent, though variable, condition following cardiac surgery and that the occurrence of postoperative delirium is associated with prolonged hospital length of stay and other negative outcomes.

\section{Methods}

\section{Study design}

The DELIRIUM-CS Canada study is a multicentre crosssectional cohort study designed to determine the incidence of delirium using a standardized delirium-screening methodology. The trial has been registered on US National Institutes of Health ClinicalTrials.gov (NCT02206880).

\section{Setting}

The DELIRIUM-CS study will be conducted in 10 centres across Canada: Calgary, Winnipeg, Hamilton, Ontario, Newmarket, Ont., Toronto, Ottawa, Montréal (2 sites), Saint John, NB, and Halifax. Over a 3-month period, all patients undergoing cardiac surgical procedures at each participating centre will be screened for postoperative delirium. Each site will have identified personnel for the collection and entry of data for the study.

The site principal investigator will be responsible for the conduct of the study and ethics approval at that site. Site communications and study documents will be sent to hospital principal investigators in addition to any nominated research personnel associated with that site.

\section{Participant selection}

\section{Inclusion criteria}

All patients undergoing cardiac surgery (coronary artery bypass grafting, valve repair or replacement, thoracic aortic procedures, cardiac transplantation and other procedures requiring cardiopulmonary bypass) who are admitted to an ICU or cardiac surgery recovery unit following their procedure will be eligible for inclusion. Patients will not be excluded on the basis of urgency or procedure type.

\section{Exclusion criteria}

Patients in whom delirium cannot be tested reliably (e.g., previous debilitating stroke, cerebral palsy, previous history of dementia, recent history of a psychotic disorder, severe hearing disability, inability to understand English or French, active seizure disorder, or Child-Pugh class B or C cirrhosis) and those with known preoperative delirium will be excluded, as will those undergoing cardiac surgery procedures that do not require admission to an ICU (e.g., pacemaker insertion, sternal débridement).

\section{Screening tools}

Each centre will decide whether to use the Intensive Care Delirium Screening Checklist (ICDSC) ${ }^{1}$ or the Confusion Assessment Method for the ICU (CAM-ICU) ${ }^{2,3}$ (Table 1) to detect delirium. Similarly, either the Riker Sedation-Agitation Scale $^{16}$ or the Richmond Agitation-Sedation Scale ${ }^{17}$ will be used to determine levels of sedation and agitation (Table 2). These 2 scales are the most valid and reliable tools for measuring the quality and depth of sedation in adult ICU patients. ${ }^{18} \mathrm{~A}$ sedation-agitation score will be paired with each delirium screening assessment; their equivalence in psychometric tools will allow pooling of sedation data. ${ }^{19}$ Simultaneous sedation assessments are important to differentiate delirium assessments confounded by sedation level from delirium independent of concomitant sedation, as sedation-associated delirium confers a different prognosis. ${ }^{20,21}$

At each centre, the ICU team has undergone formalized training in use of the ICDSC or CAM-ICU. ${ }^{22}$ Delirium screening should be performed at least every 12 hours and should be performed concomitantly with assessment of levels of sedation and agitation with the Riker Sedation-Agitation Scale or the Richmond Agitation-Sedation Scale.

\section{Data sources and collection}

Data will be extracted prospectively from patient clinical records for the cardiac surgery database/registry. Baseline demographic and clinical characteristics will also be captured and recorded on a case report form (Appendix 1, available at www.cmajopen.ca/ content/5/3/E565/suppl/DC1). Patient baseline data will include sex, age, date of surgery, surgical procedure, procedure urgency, ICU admission date and time, comorbidities (hypertension, diabetes, hyperlipidemia, smoking history, chronic obstructive pulmonary disease, peripheral vascular disease, previous cerebrovascular accident), EuroSCORE II score, and ICU and hospital length of stay.

Delirium screening will be conducted by trained nurses in all patients admitted postoperatively to the ICU or cardiac surgery recovery unit, starting on postoperative day 1 and continuing for a total of 7 days or until discharge from the ICU. Delirium and sedation data will be noted on a delirium assessment form (Appendix 2, available at www.cmajopen.ca/ content/5/3/E565/suppl/DC1).

Delirium episode data will include ICDSC or CAM-ICU, and Riker Sedation-Agitation Scale or Richmond AgitationSedation Scale scores, attending physician's diagnosis of delirium and whether the patient is receiving pharmacologic therapy for the episode.

As this is a cross-sectional study using data collected as part of routine clinical practice, no safety monitoring will be done. 


\section{Statistical analysis}

\section{Measured outcome}

Patients will be considered as having had postoperative delirium if at least 1 screening test gives a positive result for delirium 6 hours or more after anesthesia emergence (defined as stopping sedation in the operating room or the ICU). In addition to reporting an overall rate of delirium, we will report unadjusted and adjusted incidence rates of delirium by institution. Crude rates for delirium will be reported, with the potential of generating a risk model using multivariate regression modelling techniques to determine predictors of postoperative delirium in patients following cardiac surgery.

\section{Sample size}

As this study seeks to determine the incidence of postoperative delirium, there is no required sample size calculation. However, based on our data ${ }^{23}$ and on knowledge of surgical volumes at the other participating sites, we expect about 150 400 patients per site to be enrolled over the 3 -month study period.

\section{Data management}

Data management and analysis will be done by the St. Boniface Hospital/I.H. Asper Clinical Research Institute, Winnipeg. The principal means of data collection and data processing will be via electronic data entry at each site. Each patient will be given a site number and an anonymous unique study number. Data entered by each site will be passwordprotected, and the ability to access or change data before locking of the database will be restricted. All paper files will be archived and stored in a secure facility at each participating centre. Master log sheets with identifying details will remain confidential and will be stored securely at participating sites. No site monitoring will be performed. De-identified data will be exported to the St. Boniface Hospital/I.H. Asper Clinical Research Institute, where the data will be stored securely in locked files or password-protected electronic files with the use of the Research Electronic Data Capture platform. All data collected by the study will be kept for a minimum of 7 years or as otherwise required by regulatory authorities. Missing data and implausible values will be identified with the use of

\begin{tabular}{|lll|}
\hline Table 1: Delirium-screening tools & \\
\hline Item & $\begin{array}{c}\text { Intensive Care } \\
\text { Delirium Screening } \\
\text { Checklist }^{1}\end{array}$ & $\begin{array}{c}\text { Confusion Assessment } \\
\text { Method for the } \\
\text { Intensive Care Unit }\end{array}$ \\
\hline Onset & Single score of $\geq 4$ & $\begin{array}{l}\text { Single positive } \\
\text { screening result }\end{array}$ \\
\hline Termination & $\begin{array}{l}3 \text { consecutive } \\
\text { (i.e., over } 36 \mathrm{hr} \text { ) } \\
\text { negative assessments }\end{array}$ & $\begin{array}{l}3 \text { consecutive } \\
\text { (i.e., over } 36 \mathrm{hr} \text { ) } \\
\text { negative assessments }\end{array}$ \\
\hline
\end{tabular}

Table 2: Sedation-agitation scales

\begin{tabular}{|c|c|c|c|}
\hline \multicolumn{2}{|c|}{ Riker Sedation-Agitation Scale ${ }^{16}$} & \multicolumn{2}{|c|}{ Richmond Agitation-Sedation Scale ${ }^{17}$} \\
\hline Level & Description & Level & Description \\
\hline $\begin{array}{l}7 \text { Dangerous } \\
\text { agitation }\end{array}$ & $\begin{array}{l}\text { Pulling at endotracheal tube, trying to remove } \\
\text { catheters, climbing over bedrail, striking at } \\
\text { staff, thrashing side to side }\end{array}$ & +4 Combative & $\begin{array}{l}\text { Overtly combative, violent, immediate } \\
\text { danger to staff }\end{array}$ \\
\hline 6 Very agitated & $\begin{array}{l}\text { Requiring restraint and frequent verbal } \\
\text { reminding of limits, biting endotracheal tube }\end{array}$ & +3 Very agitated & $\begin{array}{l}\text { Pulls or removes tube(s) or catheter(s); } \\
\text { aggressive }\end{array}$ \\
\hline 5 Agitated & $\begin{array}{l}\text { Anxious or physically agitated, calms to verbal } \\
\text { instructions }\end{array}$ & +2 Agitated & $\begin{array}{l}\text { Frequent nonpurposeful movement, fights } \\
\text { ventilator }\end{array}$ \\
\hline $\begin{array}{l}4 \text { Calm and } \\
\text { cooperative }\end{array}$ & Calm, easily arousable, follows commands & +1 Restless & $\begin{array}{l}\text { Anxious but movements not aggressive } \\
\text { vigorous }\end{array}$ \\
\hline 3 Sedated & $\begin{array}{l}\text { Difficult to arouse but awakens to verbal stimuli } \\
\text { or gentle shaking, follows simple commands } \\
\text { but drifts off again }\end{array}$ & 0 Alert and calm & - \\
\hline 2 Very sedated & $\begin{array}{l}\text { Arouses to physical stimuli but does not } \\
\text { communicate or follow commands, may move } \\
\text { spontaneously }\end{array}$ & -1 Drowsy & $\begin{array}{l}\text { Not fully alert, but has sustained } \\
\text { awakening (eye opening/eye contact) to } \\
\text { voice ( } \geq 10 \mathrm{~s} \text { ) }\end{array}$ \\
\hline \multirow[t]{4}{*}{1 Unarousable } & \multirow[t]{4}{*}{$\begin{array}{l}\text { Minimal or no response to noxious stimuli, } \\
\text { does not communicate or follow commands }\end{array}$} & -2 Light sedation & $\begin{array}{l}\text { Briefly awakens with eye contact to voice } \\
(<10 \mathrm{~s})\end{array}$ \\
\hline & & $\begin{array}{l}-3 \text { Moderate } \\
\text { sedation }\end{array}$ & $\begin{array}{l}\text { Movement or eye opening to voice (but } \\
\text { no eye contact) }\end{array}$ \\
\hline & & -4 Deep sedation & $\begin{array}{l}\text { No response to voice, but movement or } \\
\text { eye opening to physical stimulation }\end{array}$ \\
\hline & & -5 Unarousable & $\begin{array}{l}\text { No response to voice or physical } \\
\text { stimulation }\end{array}$ \\
\hline
\end{tabular}


predetermined objective criteria. The data-cleaning process will be overseen by the DELIRIUM-CS Canada management committee.

\section{Trial monitoring and oversight}

The DELIRIUM-CS Canada management committee is responsible for all aspects of the study design and management, data analysis and publication of results.

\section{Regulatory requirements}

This study is low risk and will not affect patient management in any way. Delirium screening is considered an accreditation standard in Canadian ICUs ${ }^{24}$ and is performed routinely as part of daily assessments. To protect patient privacy, information identifying individual patients will be kept confidential at each site and will be stored securely. This will allow data queries to be addressed, which is essential to ensure data integrity, but this information will not be sent to the St. Boniface Hospital/I.H. Asper Clinical Research Institute.

Participating ICUs are involved voluntarily, and implied consent is indicated by their agreement to participate. A memorandum of understanding, if necessary, will be signed between the participating sites. Each participating site will enter a data-sharing agreement with the lead author's centre.

\section{Knowledge translation}

We will use the Strengthening the Reporting of Observational Studies in Epidemiology (STROBE) statement ${ }^{25}$ as a guideline for reporting this observational study. Data collected will be presented at the annual scientific meeting of the Canadian Cardiovascular Critical Care Society Investigator Group, scientific meetings of the Canadian Critical Care Trials Group and other forums as deemed appropriate. Data will subsequently be used for scientific publications in academic journals.

\section{Ethics approval}

This study has been approved by the University of Manitoba Research Ethics Board as well as the research ethics board at each participating centre. The study will be conducted in accordance with the International Council for Harmonisation and Good Clinical Practice principles.

\section{Interpretation}

This study will report on incidence rates of new delirium following cardiac surgery across 10 Canadian centres using standardized screening methodologies. Data from high-volume centres, both academic and community, will be collected for consecutive patients over the same period, thereby quantifying the magnitude of delirium in a large contemporary sample of the population. This is the first critical step in addressing the prevention and treatment of this life-threatening complication. Second, the study will provide rates of delirium detection using bedside personnel, providing a valuable insight into the "real-world" detectable burden of delirium. Although a reference standard for clinical evaluation of delirium is not feasible for this study, the use of 2 validated tools will allow for comparison of rates between sites. We expect that the recruitment volume at each site will enable comparisons between sites using either tool, since the variability in incidence rates of delirium with either tool over multiple studies differs. ${ }^{26}$ This may, however, result in underreporting of the actual delirium rates. Nonetheless, each site will have undergone extensive training of bedside personnel using implementation strategies shown to be effective in delirium detection. ${ }^{22}$

\section{Limitations}

We have attempted to address potential sources of bias that may arise given the observational nature of our study. To mitigate the effects of participant selection bias, patients will not be excluded on the basis of urgency or procedure type. At the institution level, we will compare incidence rates of postoperative delirium between both academic and nonacademic participating sites. A potential limitation that must be considered is how to handle data generated from centres with a low screening rate (and perhaps a screening rate that is too high). On completion of data collection for all reporting centres, we will need to consider a threshold below which the centre's data will not be included. This may provide the opportunity to make a novel contribution in that we may able to test approaches to implementation of screening tools in each institution. Indeed, it may be that some centres may be better than others or may show improvement over the 3 months of data collection. We will consider the use of a sensitivity analysis to examine how predictors change with the inclusion and exclusion of certain sites with either high or low screening rates. The variability in the reporting may, in and of itself, be quite revealing. This may offer an opportunity to look at barriers to implementation of delirium screening in a more qualitative manner and open the door to a mixed-methods analysis. Although screening for delirium is an accreditation standard in Canadian ICUs, in a mixed-method analysis, we would assess rates of compliance with screening and identify potential barriers to implementation of screening. ${ }^{27}$ Both screening tools that will be used in this study are binary in identifying delirium; neither permits an evaluation of delirium severity. Indeed, tools to assess delirium severity such as the Delirium Rating Scale ${ }^{28}$ require verbal communication, an impractical requirement in mechanically ventilated patients or those having just undergone cardiac surgery. Moreover, no delirium severity tool has been validated in critically ill patients. ${ }^{29} \mathrm{We}$ will measure duration of delirium (total number of deliriumpositive days with either tool, as opposed to a binary [ever/ never] consideration) to test whether it reflects delirium burden.

\section{Conclusion}

The DELIRIUM-CS Canada multicentre cross-sectional study proposes to determine the true incidence of delirium among Canadian patients who have undergone cardiac surgery using a standardized delirium screening methodology, as a first step in creating a national prevention/treatment clinical pathway for patients undergoing cardiac surgery. 


\section{References}

1. Bergeron N, Dubois MJ, Dumont M, et al. Intensive Care Delirium Screening Checklist: evaluation of a new screening tool. Intensive Care Med 2001;27: 859-64.

2. Ely EW, Margolin R, Francis J, et al. Evaluation of delirium in critically ill patients: validation of the Confusion Assessment Method for the Intensive Care Unit (CAM-ICU). Crit Care Med 2001;29:1370-9.

3. Inouye SK, Bogardus ST, Charpentier PA, et al. A multicomponent intervention to prevent delirium in hospitalized older patients. N Engl 7 Med 1999;340: 669-76.

4. Kornfeld DS, Heller SS, Frank KA, et al. Personality and psychological factors in postcardiotomy delirium. Arch Gen Psychiatry 1974;31:249-53.

5. van der Mast RC, Roest FH. Delirium after cardiac surgery: a critical review. 7 Psychosom Res 1996;41:13-30.

6. Rudolph JL, Jones RN, Levkoff SE, et al. Derivation and validation of a preoperative prediction rule for delirium after cardiac surgery. Circulation 2009;119: 229-36.

7. Koster S, Hensens AG, Schuurmans MJ, et al. Risk factors of delirium after cardiac surgery: a systematic review. Eur 7 Cardiovasc Nurs 2011;10:197-204.

8. Koster S, Hensens AG, Schuurmans MJ, et al. Consequences of delirium after cardiac operations. Ann Thorac Surg 2012;93:705-11

9. Loponen P, Luther M, Wistbacka JO, et al. Postoperative delirium and health related quality of life after coronary artery bypass grafting. Scand Cardiovasc 7 2008;42:337-44.

10. Martin BJ, Buth KJ, Arora RC, et al. Delirium: a cause for concern beyond the immediate postoperative period. Ann Thorac Surg 2012;93:1114-20.

11. Gottesman RF, Grega MA, Bailey MM, et al. Delirium after coronary artery bypass graft surgery and late mortality. Ann Neurol 2010;67:338-44.

12. Saczynski JS, Marcantonio ER, Quach L, et al. Cognitive trajectories after postoperative delirium. NEngl F Med 2012;367:30-9.

13. Sockalingam S, Parekh N, Bogoch II, et al. Delirium in the postoperative cardiac patient: a review. 7 Card Surg 2005;20:560-7.

14. Arora RC, Djaiani G, Rudolph JL. Detection, prevention, and management of delirium in the critically ill cardiac patient and patients who undergo cardiac procedures. Can 7 Cardiol 2017;33:80-7.

15. Ferguson TB, Hammill BG, Peterson ED, et al. A decade of change - risk profiles and outcomes for isolated coronary artery bypass grafting procedures, 1990-1999: a report from the STS National Database Committee and the Duke Clinical Research Institute. Ann Thorac Surg 2002;73:480-9.

16. Riker RR, Picard JT, Fraser GL. Prospective evaluation of the SedationAgitation Scale for adult critically ill patients. Crit Care Med 1999;27:1325-9.

17. Sessler CN, Gosnell MS, Grap MJ, et al. The Richmond Agitation-Sedation Scale: validity and reliability in adult intensive care unit patients. Am 7 Respir Crit Care Med 2002;166:1338-44.

18. Khan BA, Guzman O, Campbell NL, et al. Comparison and agreement between the Richmond Agitation-Sedation Scale and the Riker SedationAgitation Scale in evaluating patients' eligibility for delirium assessment in the ICU. Chest 2012;142:48-54.

19. Mehta S, Burry L, Cook D, et al. Daily sedation interruption in mechanically ventilated critically ill patients cared for with a sedation protocol: a randomized controlled trial. FAMA 2012;308:1985-92.

20. American Psychiatric Association. Diagnostic and statistical manual of mental disorders, 5th edition. Arlington (VA): American Psychiatric Association Publishing; 2013.

21. Patel SB, Poston JT, Pohlman A, et al. Rapidly reversible, sedation-related delirium versus persistent delirium in the intensive care unit. Am $\mathcal{F}$ Respir Crit Care Med 2014;189:658-65.
22. Devlin JW, Marquis F, Riker RR, et al. Combined didactic and scenario-based education improves the ability of intensive care unit staff to recognize delirium at the bedside. Crit Care 2008;12:R19.

23. Arenson BG, MacDonald LA, Grocott HP, et al. Effect of intensive care unit environment on in-hospital delirium after cardiac surgery. 7 Thorac Cardiovasc Surg 2013;146:172-8.

24. Standard 10.9. Ottawa: Accreditation Canada; 2013. Available: www.accreditation .ca/en/ (accessed 2015 Nov. 1).

25. Vandenbroucke JP, von Elm E, Altman DG, et al. Strengthening the Reporting of Observational Studies in Epidemiology (STROBE): explanation and elaboration. Int 7 Surg 2014;12:1500-24.

26. Devlin JW, Fraser GL, Joffe AM, et al. The accurate recognition of delirium in the ICU: The emperor's new clothes? Intensive Care Med 2013;39:2196-9.

27. Nishimura K, Yokoyama K, Yamauchi N, et al. Sensitivity and specificity of the Confusion Assessment Method for the Intensive Care Unit (CAM-ICU) and the Intensive Care Delirium Screening Checklist (ICDSC) for detecting post-cardiac surgery delirium: a single-center study in Japan. Heart Lung 2016;45:15-20.

28. De J, Wand APF. Delirium screening: a systematic review of delirium screening tools in hospitalized patients. Gerontologist 2015;55:1079-99.

29. Barr J, Fraser GL, Puntillo K, et al. Clinical practice guidelines for the management of pain, agitation, and delirium in adult patients in the intensive care unit. Crit Care Med 2013;41:263-306.

DELIRIUM-CS Investigators: Alan Sobey MD (Edmonton, Alta.); Barry Kushner PharmD, Philippe Couillard MD, Andre Ferland MD (Calgary, Alta.); Rakesh C. Arora MD PhD (lead/corresponding author), Kelsey Uminski BSc, Navdeep Tangri MD PhD, Hilary Grocott MD, Brett Hiebert MSc (Winnipeg, Man.); Dave Nagpal MD, (London, Ont.); Alison Fox-Robichaud MD (Hamilton, Ont.); Lisa Hutchinson MD (Newmarket, Ont.); Christopher Gabor MSc (Hamilton, Ont.); George Djainai MD, Angela Jerath MD (Toronto, Ont.); Bernard McDonald MD PhD (Ottawa, Ont.); Yoanna Skrobik MD, Sheldon Magder MD, Yoan Lamarche MD MSc, Andre Denault MD PhD (Montréal, Que.); Ansar Hassan MD PhD (Saint John, NB); Jean-François Légaré MD MSc (Halifax, NS).

Contributors: This study will be published under the authorship of the Canadian Cardiovascular Critical Care Society (CANCARE) Investigator Group investigators with the writing committee listed as primary authors. This is a collaborative project between a research initiative developed with CANCARE and the Canadian Critical Care Trials Group. As such, this paper was submitted on behalf of the DELIRIUM-CS investigators on behalf of the CANCARE Investigator Group and the Canadian Critical Care Trials Group.

Funding: This study is funded by a Meeting and Planning Grant (KPE126811) from the Canadian Institutes of Health Research. The St. Boniface Hospital/I.H. Asper Clinical Research Institute will fund data management, operations and analysis. No site payments are available at the time of writing, but funding will be sought.

Supplemental information: For reviewer comments and the original submission of this manuscript, please see www.cmajopen.ca/content/5/3/ E565/suppl/DC1. 